\title{
Presente y futuro de la Cooperación al Desarrollo: Entre el debate de la eficacia y las dudas sobre su pertinencia
}

\author{
KOLDO UNCETA SATRUSTEGUI \\ Instituto Hegoa, UNIVERSIDAD DEL PAÍS VASCO (UPV/EHU), ESPAÑA. E-mail: \\ koldo.unceta@ehu.es
}

\begin{abstract}
RESUMEN
El presente artículo presenta un panorama general sobre los principales debates que afectan al presente y al futuro de la cooperación para el desarrollo y su relación con la crisis, enmarcando de esa manera los distintos trabajos que se presentan en este número de la Revista de Estudios de Economía Aplicada. Dichos debates se encuentran asociados por una parte a la amplia literatura surgida a lo largo de los últimos veinte años sobre la eficacia de la cooperación y la necesidad de mejorar el sistema de ayuda para incrementar su calidad. Y por otra parte, se relacionan con los puntos de vista que, desde una perspectiva más de fondo, vienen a cuestionar la propia identidad de la cooperación en el momento presente y el papel que la misma puede desempeñar en los próximos años.
\end{abstract}

Palabras clave: Cooperación para el Desarrollo, AOD, desarrollo, eficacia ayuda, crisis económica.

\section{Present and Future of Development Cooperation: Between the Discussion of the Effectiveness and Doubts About its Relevance}

\begin{abstract}
This article presents an overview of the most important debates that explain not only the present and the future of development co-operation, but also its relation to the crisis, framing in within the papers published in the current issue of the Journal of Applied Economics Studies. On one hand, these debates are associated to the vast literature emerged over the last twenty years concerning the effectiveness of aid and the need of an improvement of its quality. On the other hand, they are related to a wider viewpoint that questions the identity of the co-operation nowadays and the role that it can play in the following years.
\end{abstract}

Keywords: Development Cooperation, ODA, Development, Aid Effectiveness, Economic Crisis.

Clasificación JEL: O10, O19, F35

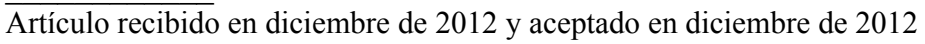

Artículo disponible en versión electrónica en la página www.revista-eea.net, ref. ə-30314 
La cooperación al desarrollo, surgida tras la segunda guerra mundial en condiciones bien diferentes a las que existen hoy en día en el mundo, se encuentra en estos momentos sometida a importantes debates que constituyen el reflejo de la doble crisis que existe en su seno. Por un lado la que afecta a lo que se conoce como el Sistema de Ayuda, a través del cual se canalizan los flujos de AOD, sistema que en la actualidad se ve sometido a importantes tensiones derivadas tanto de las dudas existentes sobre la eficacia, como de la menor disponibilidad de fondos aportados por diversos países aquejados por la crisis financiera. Y, por otra parte, la que tiene que ver con la propia identidad de la cooperación y su relación con los retos del desarrollo a comienzos de este siglo XXI, cuestión que enlaza con las discusiones sobre los cambios operados en las relaciones económicas internacionales a lo largo de las últimas décadas y el impacto que todo ello ha tenido sobre los procesos y las dinámicas de desarrollo en unos y otros países.

\section{LA EVOLUCIÓN DEL SISTEMA DE AYUDA Y LA CUESTIÓN DE LA EFICACIA}

El primero de estos dos asuntos ha estado presente en la agenda de debates sobre la cooperación desde mediados de la década de los 90, cuando comenzó a instalarse cierta preocupación sobre la ausencia de resultados y se hizo presente la idea de la "fatiga de la ayuda". La consecuencia de todo ello fue la apertura de un vivo debate, algunas de cuyas preguntas quedaron planteadas por el Banco Mundial (World Bank, 1998), debate que daría lugar a una amplia literatura sobre la eficacia de la ayuda y su evaluación planteada desde muy diversos puntos de vista (Cassen, 1994, Alonso y Mosley 1999, Burnside y Dollar 2000, Vandemoortele 2002, Dalgaard, Hansen y Tarp 2004, Easterly 2008, Kabeer 2010, Craviotto y Antolin 2009, Martinez y Sanahuja 2009). La cuestión de la eficacia ha ocupado asimismo un importante papel en la mayor parte de los foros celebrados desde el comienzo del nuevo siglo. En este sentido, Roma, París, Accra, o Busan representan eslabones de una larga cadena de discusiones, propuestas, y acuerdos diversos, orientados a aumentar la calidad y la eficacia de la Ayuda.

Sin embargo, durante todo este tiempo han sido muchas las voces que han señalado la necesidad de ampliar el campo del debate sobre la eficacia de la cooperación al desarrollo, llevándolo más allá de los estrechos márgenes de la cadena de la Ayuda. Algunos de los trabajos anteriormente mencionados, y otros, han incidido sobre este asunto. En este orden de cosas, los principios de París aparecen como una referencia de interés para mejorar la definición y gestión de gestión de algunos programas de cooperación, pero insuficientes para dotar al sistema de una eficacia que se ve limitada por elementos diversos que se sitúan fuera de dicha cadena. La cuestión de la coherencia de políticas ha 
sido citada de manera recurrente como uno de los grandes retos que condicionan la eficacia (Alonso y Fitzgerald 2003, Olivié y Sorroza 2006, Castermans y Heintze 2007, Roodman y Prieto 2008). Otras voces han subrayado la necesidad de repensar los propios instrumentos de financiación del desarrollo que vayan más allá del sistema de la AOD e incluyan el debate sobre la fiscalidad internacional, o la provisión de Bienes Públicos Globales (Alonso 2009, Severino y Ray 2009). En este contexto, muchos de los problemas suscitados tienen que ver con la gobernanza del sistema de ayuda, pero al mismo tiempo superan ampliamente el ámbito del mismo. La descoordinación entre políticas, que en muchos casos lleva a actuaciones abiertamente contradictorias con los objetivos de desarrollo planteados, constituye una limitación que, de no superarse, amenaza con echar por tierra cualquier resultado que pudiera esperarse de la AOD y de las propuestas formuladas para incrementar su eficacia. Por ello, el debate sobre este asunto requiere de un enfoque más amplio que contemple un abanico diverso de temas relacionados son la coordinación de políticas de desarrollo, con la reorganización del sistema financiero internacional, y con la gobernanza global.

En los últimos años, las dudas sobre el sistema de Ayuda se han visto ampliadas como consecuencia de la crisis financiera que ha venido afectando a algunos países que, como España, más habían incrementado sus aportaciones para Ayuda al Desarrollo. Aun es pronto para valorar el impacto de todo ello sobre la evolución futura de los fondos de AOD ya que tras el descenso operado en 2008-2009, se produjo una ligera recuperación en 2010 a la que siguió un nuevo retroceso en 2011. Sin embargo, más allá de las cifras concretas, la importancia del impacto de la crisis en las economías occidentales, unida al nuevo papel que están asumiendo los llamados países emergentes, hace que el examen sobre la orientación de la cooperación al desarrollo vaya a estar mediatizado en los próximos años por la profundidad de los cambios que se están produciendo en la jerarquía financiera internacional y en el papel que unos y otros países puedan desempeñar en el futuro.

Desde una perspectiva más doméstica, la AOD española ya se ha visto fuertemente afectada por la crisis, hasta el punto de haber sufrido un importante retroceso en los fondos aportados, tanto en términos absolutos como en relación con el PIB. Dicho retroceso se ha puesto de manifiesto en todos los ámbitos, desde el nivel central, hasta el autonómico y municipal. Este último aspecto amenaza por otra parte con acabar con una de las señas de identidad más características de la cooperación al desarrollo española, como ha sido el auge alcanzado por la cooperación descentralizada, apoyada desde CC. AA., Ayuntamientos y otras entidades locales mediante significativas aportaciones financieras. El hecho de que los fondos aportados desde estas administraciones hayan llegado a representar en torno al 15\% del total de la AOD española cons- 
tituye un caso excepcional en Europa, que en las actuales circunstancias se encuentra claramente amenazado.

Las cuestiones más arriba señaladas son algunos de los elementos que conforman el debate sobre la agenda futura de la cooperación. En cualquier caso, debe subrayarse que se trata de una agenda en la que con demasiada frecuencia tiende a identificarse el sistema de ayuda con el conjunto del sistema de cooperación, cuando en realidad debería ser considerado como una parte del mismo. Esta reflexión conduce directamente a la segunda cuestión que concita especialmente la atención de los investigadores y de algunos organismos internacionales, y que no es otra que la relativa al futuro papel de la cooperación, a la propia identidad de la misma, en un contexto de profundos cambios en la esfera internacional y en lo que han venido a llamarse las relaciones Norte/Sur. En este sentido, las iniciales discusiones sobre la manera de aumentar la eficacia de la ayuda, han acabado por afectar al debate sobre su propia pertinencia.

\section{LOS CAMBIOS EN LA ESFERA INTERNACIONAL, Y EL SIGNIFICADO Y PERTINENCIA DE LA COOPERACIÓN AL DESARROLLO}

Hasta hace apenas tres décadas, las condiciones en las que se desenvolvía la vida de las personas dependían en buena medida de la capacidad de las instituciones, organizaciones, y grupos sociales de cada país de promover acciones que pudieran incrementar el bienestar de la gente. Los gobiernos, las empresas, o las organizaciones sociales del más diverso signo, desarrollaban sus acciones en un marco determinado en lo fundamental por reglas establecidas al interior de cada país. En el caso de los países más desfavorecidos, la cooperación al desarrollo, u otros instrumentos de cooperación económica o financiera debían servir para posibilitar que los mismos pudieran acelerar su propio desarrollo sin que ello cuestionara, al menos formalmente, su capacidad de avanzar en una u otra dirección, y establecer sus propias políticas económicas y sus propias normas de organización social.

Hoy, por el contrario, las condiciones del proceso de globalización -y muy especialmente las relativas a la liberalización económica y financiera llevada a cabo- dibujan un panorama en el que los precios de los bienes y servicios, los salarios, los tipos de interés, los impuestos, las inversiones, o el gasto público, vienen determinados en gran medida por factores exógenos, propios del marco global en el que se desenvuelven las actividades económicas. Y, en ese contexto, las instituciones y organizaciones sociales de los más diversos países se ven enfrentadas a problemas globales, y obligadas por tanto a tener en cuenta los mismos a la hora de concebir y desarrollar sus propias estrategias de desarrollo. 
En el nuevo escenario surgido, la globalización ha propiciado un cambio radical de las condiciones en que venía desenvolviéndose la financiación del desarrollo, como consecuencia del impacto de la libre circulación de capitales, y de la consiguiente dificultad de orientar la inversión, a través de políticas públicas, hacia el logro de determinados objetivos de desarrollo. Ello se encuentra, además, relacionado con la vulnerabilidad que, para muchos países y muchos procesos de desarrollo, supone la incertidumbre asociada al actual funcionamiento de los mercados financieros internacionales. Por otra parte, la globalización ha supuesto la emergencia de problemas globales de desarrollo que trascienden las fronteras nacionales y que ya no pueden ser tratados, únicamente, en el marco de los Estados. Las cuestiones medioambientales se encuentran seguramente entre las más importantes a este respecto, pero no son las únicas, ya que a ellas hay que sumar las derivadas de los movimientos migratorios, de las crisis financieras, o de la expansión transnacional de algunas enfermedades.

En estas condiciones, se ha ido produciendo, poco a poco, una creciente internacionalización, no sólo de los problemas que afectan al desarrollo, sino también de las preocupaciones y las estrategias de instituciones y organizaciones de unos y otros países, cuestión que afecta de lleno a la cooperación internacional en sus diversas vertientes y también, específicamente, a la cooperación al desarrollo. En consecuencia, ésta no puede ser ya concebida por más tiempo como mera transferencia de recursos técnicos y financieros, necesitando ser contemplada como un conjunto de medidas que afectan a unos y otros países, y orientadas a que el progreso humano sea posible y viable a escala mundial.

Otro de los fenómenos que más han influido en la percepción de la cooperación a lo largo de los últimos años es el referido a los cambios operados en la concepción del desarrollo y muy especialmente en la ruptura del consenso existente en otro tiempo sobre la necesidad de un compromiso explícito con el desarrollo por parte de las instituciones públicas -tanto en el ámbito nacional como internacional-, y la sustitución de dicho consenso por la hegemonía de un nuevo pensamiento favorable a que el mercado sea el encargado de asignar los recursos y lograr una mayor eficiencia económica que, supuestamente, habría de traducirse en mayores cotas de progreso. En línea con este diagnóstico, el pensamiento oficial ha pasado de considerar el desarrollo como una responsabilidad de los poderes públicos y los organismos multilaterales, a una interpretación del mismo en la que la liberalización económica y el progresivo desmantelamiento del Estado constituyen las principales señas de identidad (Unceta y Arrinda, 2009). Y, como corolario de lo anterior, de una concepción de la AOD basada en el fortalecimiento de las políticas de desarrollo, se ha ido pasando a otra en la que la acción humanitaria o paliativa ocupa cada vez mayores esfuerzos, todo lo cual ha trastocado, en buena medida, tanto los instrumentos puestos en juego, como el papel que los distintos agentes desempeñan en la tarea de la cooperación. 
La crisis que estalló en 2008 refleja en buena medida algunos de los problemas propiciados por las transformaciones habidas en las últimas décadas, pero al mismo tiempo constituye la expresión de un fenómeno que ha contribuido a acelerar de forma muy acusada algunas manifestaciones de dichos cambios. Una de las más significativas es precisamente la que afecta a la consideración de las relaciones Norte/Sur y a lo que algunos han denominado como disolución de la metáfora jerárquica ordenadora de las relaciones internacionales (Dominguez, 2011). Dichas relaciones se han visto alteradas notablemente como consecuencia de diversos fenómenos -entre los que destacan el papel de nuevos actores como China, India o Brasil-, y como resultado del distinto impacto de la crisis en unos y otros países. El hecho de que un buen número de países asiáticos, africanos y latinoamericanos registren elevadas tasas de crecimiento, en tanto muchos países occidentales se enfrentan un débil crecimiento cuando no a la recesión, representa mejor que ninguna otra, la imagen de un mundo en el que las categorías que sustentaron el surgimiento de la cooperación ya no son de utilidad para comprender los fenómenos actuales.

En estas circunstancias, la cooperación al desarrollo se enfrenta a una importante crisis de identidad, que cuestiona su propio papel en un mundo en el que los nuevos problemas emergentes requieren de otros enfoques e instrumentos para enfrentarlos. Ello se refleja en diversos trabajos que plantean la necesidad de nuevos diagnósticos y que subrayan distintos aspectos de la crisis de la cooperación (Sobhan 2006, Unceta y Arrinda 2009, Sörensen 2010, Alonso y Ocampo 2012). Algunos autores han puesto de manifiesto cambiar incluso el propio término de AOD (Severino y Ray 2009), en la búsqueda de un nuevo paradigma que responda más cabalmente a las necesidades actuales de financiación del desarrollo.

\section{UNA REFLEXIÓN IMPRESCINDIBLE}

Estas y otras cuestiones que están presentes en el debate forman parte de las preocupaciones suscitadas en los trabajos que se publican en este número de la Revista de Estudios de Economía Aplicada dedicado a la Cooperación al Desarrollo frente a los retos de la Economía Global. En ellos se ofrece un variado panorama de temas que abarcan distintas perspectivas sobre dicho debate. Por un lado, se presentan trabajos más generales que reflexionan sobre el papel de la cooperación en el marco del sistema económico mundial (Martinez Peinado y Maestro). Por otra parte, un par de trabajos se centran en el estudio del vínculo establecido por algunas instituciones entre comercio y cooperación al desarrollo como parte de su estrategia, como es el caso de la UE (Bidaurratzaga y Zabalo), o en el seno de la iniciativa de Ayuda para el Comercio de la OMC (Rueda y Gozalo). Otros artículos examinan la política de algunos actores específicos, como por ejemplo la cooperación llevada a cabo por el gobierno español y su 
especialización (Larrú y Tezanos). La Universidad como espacio de trabajo merece también un análisis específico, centrado en este caso en el ámbito de la educación al desarrollo (Ortega, Cordón y Sianes). Finalmente, diversas contribuciones estudian enfoques transversales, sectoriales, o aspectos específicos de las políticas de cooperación, tratando de evaluar los mismos. Es el caso del análisis sobre el enfoque de género en las políticas de cooperación descentralizadas (Zabala, Martinez y Labaien), de la aproximación al papel de la tecnología en las estrategias de Desarrollo Humano (Fernandez Baldor, Boni y Hueso), del estudio sobre la cooperación española para el fortalecimiento institucional (Bandeira y Warleta), o del análisis sobre la AOD y los servicios sociales básicos (Muñoz y Torres).

Se trata en todos los casos de trabajos que abordan una problemática compleja y que se encuentra en constante evolución, lo cual convierte en imprescindibles la reflexión y el debate sobre la misma.

\section{REFERENCIAS BIBLIOGRÁFICAS}

ALONSO, J. A. y MOSLEY, P. (eds) (1999): La eficacia de la cooperación internacional al desarrollo: evaluación de la ayuda. Ed. Cívitas, Madrid.

ALONSO, J. A. y FITZGERALD, V. (eds.) (2003): Financiación del desarrollo y coherenciade políticas de los donantes. La Catarata, Madrid.

ALONSO, J. A. (dir.) (2009): Financiación del Desarrollo: viejos recursos, nuevas propuestas. Fundación Carolina- Siglo XXI. Madrid.

ALONSO, J. A. y OCAMPO, J. A. (2012). Development Cooperation in times of crisis. Columbia University Press. Nueva York.

BROAD, R. Y CAVANAGH, J. (2009). Development Redefined. How the market met its match. London: Paradigm Publishers.

BURNSIDE, C. y DOLLAR, D. (2000). "Aid, Policies, and Growth" en The American Economic Review, Vol. 90, № 4, pp. 847-868.

CASSEN, R. (1994). Does Aid Work? Oxford: Clarendon Press.

CASTERMANS, R. y HEINTZE, P. H. (2007): Policy Coherence for Development. A practical guide. EU Coherence - Evert Vermeer Foundation. Noviembre. Países Bajos.

CRAVIOTTO, N. y ANTOLIN, L. (coords.) (2009): La movilización por la igualdad de género y la eficacia de la ayuda. Fundación Carolina-WIDE. Documento de trabajo $n^{\circ} 36$. Madrid.

DOMINGUEZ, R. (2011): La crisis e identidad del Sistema de Ayuda. Fundación Carolina. http://www.fundacioncarolina.es/es-ES/nombrespropios/Documents/ NPDom\%C3\%ADnguez1105.pdf 
EASTERLY, W. (2008). Reinventing Foreing Aid. Cambridge, Massachusetts: MIT Press.

MARTINEZ, I. y SANAHUJA, J. A. (2009): La agenda internacional de la eficacia de la ayuda y la cooperación descentralizada en España. Fundación Carolina. Documento de trabajo $\mathrm{n}^{\circ} 38$. Madrid.

OLIVIE, I. y SORROZA, A. (2006): Más allá de la Ayuda: Coherencia de políticas económicas para el desarrollo. Real Instituto Elcano, Ariel.

ROODMAN, D. y PRIETO, C. (2008) Índice de Compromiso con el Desarrollo (CDI,Commitment to Development Index), Center for Global Development. España. www.cgdev.org/cdi

SEVERINO, J-M. y RAY, O. (2009). "The End of ODA: Death and Rebirth or a Global Public Policy”. Center for Global Development Working Paper, $n^{\circ} 167$ (March).

SOBHAN, R. (2006). "Challenging the injustice of poverty: rethinking aid strategies" en Steen Folke and Henrik Nielsen (Eds.). Aid impact and poverty reduction. New York: Palgrave-Macmillan.

SÖRENSEN, J. S. (2010). Challenging the Aid Paradigm. Western Currents and Asian Alternatives. London: Palgrave Macmillan.

UNCETA, K. y ARRINDA, A. (Eds.): Development cooperation. Facing the challenges of global change. Current Researdch $n^{\circ} 3$. University of Nevada. Reno

VANDEMOORTELE, J. (2002). Are we really reducing global poverty? United Nations Development Programme. Bureau for Development Policy.

WORLD BANK (1998). Assessing Aid: What Works, What Doesn't, and Why. Oxford University Press. 